\title{
Utility of hyperchromic cell percentage, flags, and red cell cytograms generated by Advia-120 hematology analyzer as a potential screening tool in hereditary spherocytosis
}

\author{
Sugandha ${ }^{1} \cdot$ Naveen Kakkar $^{2}$ (D) $\cdot$ M. Joseph John ${ }^{3}$ \\ Received: 19 August 2020 / Accepted: 12 October 2020 / Published online: 19 October 2020 \\ (C) Springer-Verlag GmbH Germany, part of Springer Nature 2020
}

\begin{abstract}
Hereditary spherocytosis (HS) is the commonest inherited red cell membrane disorder seen in clinical practice. Modern hematology analyzers generate newer parameters that can help in screening of HS. The present study focuses on the utility of red blood cell data generated by Advia-120 hematology analyzer as a screening tool for patients with HS. Blood samples from 21 patients with HS were analyzed on Advia-120 (Technicon $\mathrm{H}^{*} 1$ series) analyzer. The percentage of hyperchromic cells and hyperchromic and hemoglobin concentration variance flags along with the RBC cytogram were studied. A control group of 50 healthy individuals and 50 patients with other hematological disorders was also studied. Osmotic fragility test was done in all patients with suspected HS. All hematological parameters including percentage of hyperchromic red cells were compared between the study group, healthy controls, and other hematological disorders. Twenty of the 21 patients with HS were anemic. Peripheral blood smear in all patients showed variable numbers of spherocytes (5-50\%). Osmotic fragility test was positive in all patients. The mean percentage of hyperchromic cells in patients with HS-18.2 $\pm 13.0 \%$ was significantly higher $(p<0.0001)$ than that seen in the control group and in patients with other hematological disorders. Hyperchromic RBC $>6 \%$ showed a sensitivity of $100 \%$ and specificity of $99 \%$ for the diagnosis of HS. All patients showed flagging for hyperchromic cells. The RBC cytograms in all 21 patients showed significant number of RBCs plotted in the hyperchromic zone. The percentage of hyperchromic cells, hyperchromic cell flagging, and RBC cytogram pattern generated by Advia-120 hematology analyzer are useful tools in the screening of patients with suspected HS.
\end{abstract}

Keywords Hereditary spherocytosis $\cdot$ Automated $\cdot$ Hyperchromic cells $\cdot$ Flag $\cdot$ Red cells

\section{Introduction}

Hereditary spherocytosis (HS) is the most common inherited red cell membrane disorder seen in clinical practice with a prevalence of 1 in 5000 to 1 in 2000 in the Caucasian population [1]. It is clinically heterogeneous with patients being

\section{Naveen Kakkar}

kakkar.naveen@gmail.com

1 Department of Pathology, Vardhman Mahavir Medical College \& Safdarjung Hospital, New Delhi, India

2 Department of Pathology, Maharishi Makandeshwar Medical College \& Hospital, Kumarhatti, Solan, Himachal Pradesh 173229 , India

3 Department of Clinical Hematology, Hemato-oncology and Bone Marrow (Stem cell) Transplantation, Christian Medical College \& Hospital, Ludhiana, Punjab, India asymptomatic or presenting with moderate to severe hemolytic anemia. Complete blood count (CBC), reticulocyte count, and osmotic fragility test (OFT) are commonly employed for its diagnosis in the background of anemia, jaundice, splenomegaly, presence of gall stones on abdominal ultrasound, and indirect hyperbilirubinemia [1-3].

The variability in the number of spherocytes seen in the peripheral blood smear makes the diagnosis difficult at times. The observer's experience also plays a crucial role in flagging the diagnosis for the clinician $[1,4,5]$. The OFT, which has been traditionally used for detecting spherocytes, is not very sensitive and also non-specific [2]. A normal OFT does not eliminate the possibility of HS [4]. Normal OFT may be seen with mild form of the disease that may not have enough spherocytes in the peripheral blood [5]. Osmotic fragility test is also time-consuming and cumbersome [2, 4]. Flow cytometric analysis with eosin-5-maleimide (EMA) labeled RBCs has high sensitivity and specificity for diagnosing HS [6]. 
Flow cytometric OFT can also be done using a red cell suspension diluted with distilled water and measuring the percentage of residual red cells [7]. Genetic studies have shown mutations in HS-related genes, SPTA1, SPTB, ANK1, SLC4A1, and EPB42. However, the absence of a hotspot mutation and heterogeneity makes it challenging to rule out HS [8]. Modern-day sophisticated automated hematology analyzers have introduced newer parameters that have potential for use as an aid in the screening of some disorders [9]. Previous studies have shown the utility of these novel parameters in the screening of HS [10-17]. The Technicon $\mathrm{H}^{*} 1$ series instruments generate newer red cell parameters related to the percentage of hyperchromic cells and hyperchromic and hemoglobin concentration variance flags [10-14]. In addition, the graphical representation of the RBC cytogram also provides clues to the presence of spherocytes. The present study focuses on the use of newer automated parameters and graphical data generated by Advia-120 analyzer as a screening tool for patients with HS.

\section{Materials and methods}

The present study was conducted in the hematology laboratory of a tertiary care hospital in North India. Blood samples from 21 patients with HS were included. Samples were drawn in di-potassium EDTA containers for complete blood count $(\mathrm{CBC})$, peripheral blood smear, and reticulocyte count. Heparinized tubes were used for OFT. Complete blood count was run in Advia-120 (Technicon $\mathrm{H}^{*} 1$ series) (Siemens) analyzer within $2 \mathrm{~h}$ of collection.

Advia-120 (Technicon $\mathrm{H}^{*} 1$ series) is a 6-part differential system that analyzes blood cells by flow scatter and enumerates the white blood cell differential count using myeloperoxidase staining. Red cell analysis is done by flow cytometry and laser. The red cells are isovolumetrically sphered before analysis. The graphical output includes histograms and a nine-grid red cell cytogram which is a graphical representation of two light scatter measurements of the red blood cells. These include high angle $\left(5-15^{\circ}\right)$ light scatter (x-axis) and low angle scatter $\left(2-3^{\circ}\right)$ scatter (y-axis). On the volume/hemoglobin concentration (V/HC) cytogram, hemoglobin concentration is plotted along the $\mathrm{x}$-axis and the cell volume along the y-axis. After isovolumetric sphering of RBCs, hemoglobin concentration markers are set at $28 \mathrm{~g} / \mathrm{dL}$ and $41 \mathrm{~g} / \mathrm{dL}$. On the y-axis, RBC volume markers are set at $60 \mathrm{fl}$ and $120 \mathrm{fl}$ (Fig. 1). Blood samples with normal red cell population show most RBCs to fall in the center of the V/HC cytogram. The instrument also quantifies hyperchromic red cells and flags the same $(+,++$, or +++$)$ indicating increasing number of hyperchromic red blood cells $[+\%$ HYPER $=4.0 \%$ to $7.9 \%,++\%$ HYPER $=8.0 \%$ to $12.0 \%,+++$ $\%$ HYPER $>12.0 \%]$. HC variance flag is triggered if the

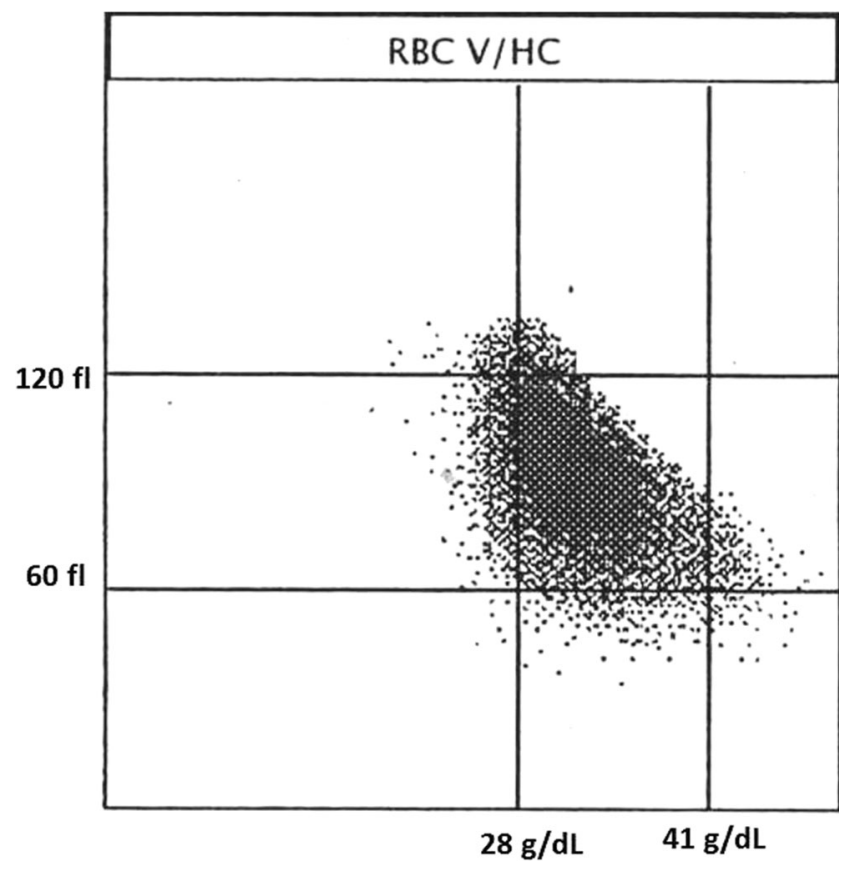

Fig. 1 Normal RBC cytogram showing division into 9 zones by a grid. The volume markers are set at $60 \mathrm{fl}$ and $120 \mathrm{fl}$, while the hemoglobin concentration markers are set at $28 \mathrm{~g} / \mathrm{dL}$ and $41 \mathrm{~g} / \mathrm{dL}$. Red cells in individuals with normocytic normochromic picture are plotted in the central zone

variation in cell hemoglobin concentration measured as hemoglobin distribution width (HDW) is equal to or greater than $3.4 \mathrm{~g} / \mathrm{dL}$. The three flagging limits are $+\mathrm{HDW} \%=3.4 \mathrm{~g} / \mathrm{dL}$ to $3.9 \mathrm{~g} / \mathrm{dL},++\mathrm{HDW} \%=4.0 \mathrm{~g} / \mathrm{dL}$ to $4.6 \mathrm{~g} / \mathrm{dL}$, and +++ HDW $\%>4.6 \mathrm{~g} / \mathrm{dL}$.

Clinical details noted in all patients included pallor, jaundice, organomegaly, and history of gall stones. The hematological parameters noted were hemoglobin $(\mathrm{Hb}), \mathrm{RBC}$ indices (MCV, MCH, MCHC, RDW), total leukocyte count (TLC), and platelet count. The percentage of hyperchromic cells, hyperchromic and hemoglobin concentration variance flags, and the graphical representation of the RBC cytogram were also studied. A control group of 50 healthy individuals was also included. To study the possibility of false-positive hyperchromic cell flagging, automated data from 50 patients with other hematological disorders (non-spherocytic) with associated anemia were also studied. These included patients with infections, thrombocytopenia, leukemia, and other hematological malignancies. Osmotic fragility test was performed in all patients with suspected HS. The diagnosis of HS was made on the basis of clinical history of anemia, recurrent jaundice, indirect hyperbilirubinemia, presence of spherocytes in the peripheral blood smear, and a positive OFT. In all patients, Direct Coombs test (DCT) was done to rule out AIHA.

Statistical analysis ANOVA was used to analyze the differences in the mean percentages of hyperchromic red cells between the study group, control, and patients with 
other hematological disorders. Pearson correlation coefficient was used to compare $\mathrm{MCHC}$ and percentage of hyperchromic cells. Free access online software (http:// www.openepi.com)-Openepi was used.

\section{Results}

Twenty-one patients with HS were included in the study. Of these, 20 patients were anemic. Of the 21 patients, 9 were icteric at the time of presentation. Splenomegaly was seen in 10 patients and hepatomegaly in only 3 patients. Mild indirect hyperbilirubinemia was seen in all patients. None of the patients in our study underwent splenectomy. The automated data showed an increase in the percentage of hyperchromic cells and hyperchromia flag in all patients. The hematological parameters and flagging data in patients with HS, control group, and patients with other hematological disorders are shown in Table 1. Except MCH, all other hematological parameters showed a significant difference between the groups. None of the patients in the control group or other hematological disorders showed a significant increase in hyperchromic cells or hyperchromia flag although few patients showed flagging for hemoglobin variance. Mean \% hyperchromic cells was significantly higher $(p<0.0001)$ in patients with HS compared to the control group and other hematological disorders (Table 1).

The peripheral blood smears in all patients showed variable number of spherocytes (5-50\%) (Fig. 2), macrocytosis and polychromasia in few cases. Reticulocytosis was seen in all cases. No autoagglutination was observed in any of the patients. Osmotic fragility test was positive in all patients with a

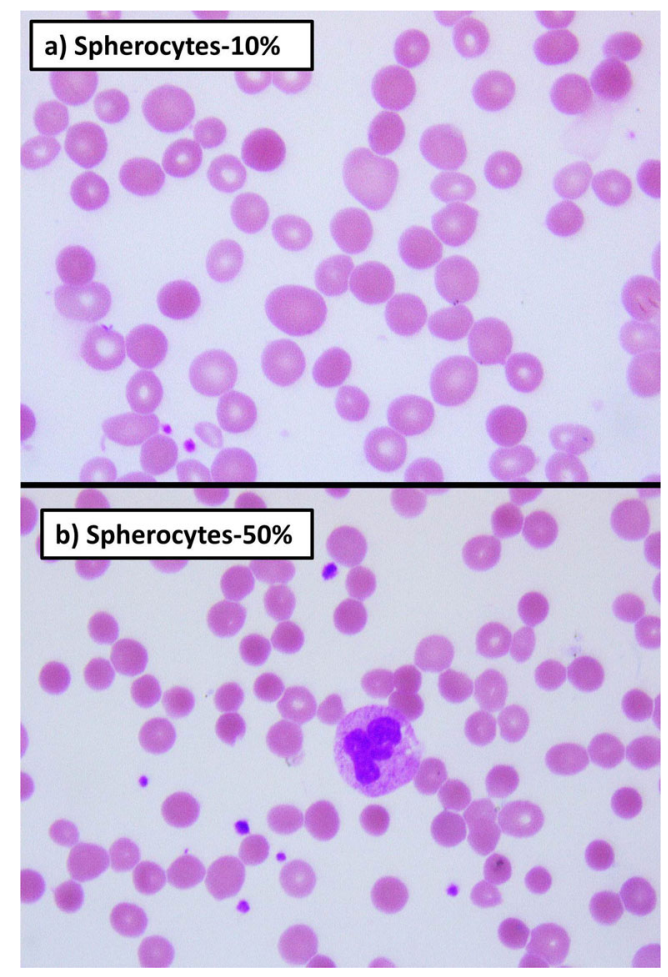

Fig. 2 Shows variable number of spherocytes in the peripheral blood smears in two patients [a 10\%, b 50\%) (Leishman $\times 1000)$

raised median corpuscular fragility greater than the control value. Direct Coombs test was negative in all patients with HS. Although 15 of the 21 patients with HS had raised $\mathrm{MCHC}$, it showed poor correlation $\left(r^{2}=0.2181\right)$ with the percentage of hyperchromic cells (Fig. 3). Hyperchromic RBC > $6 \%$ showed a sensitivity of $100 \%$ and specificity of $99 \%$ for the diagnosis of HS.

Table 1 Comparison of CBC parameters and flagging data among patients with hereditary spherocytosis $(n=21)$, healthy individuals $(n=50)$, and patients with other hematological disorders $(n=50)$

\begin{tabular}{|c|c|c|c|c|c|c|c|}
\hline \multirow[t]{2}{*}{ Parameter } & \multicolumn{2}{|c|}{ Hereditary spherocytosis } & \multicolumn{2}{|c|}{ Healthy individuals } & \multicolumn{3}{|c|}{ Other hematological disorders } \\
\hline & Mean \pm SD & Range & Mean \pm SD & Range & Mean \pm SD & Range & $p$ value \\
\hline \multicolumn{8}{|l|}{ CBC parameters } \\
\hline $\mathrm{Hb}(\mathrm{g} / \mathrm{L})$ & $94 \pm 23$ & $50-142$ & $134 \pm 10$ & $120-156$ & $97 \pm 34$ & $30-162$ & $<0.001$ \\
\hline $\mathrm{MCV}$ (fl) & $85.4 \pm 10.1$ & $72.5-111.1$ & $87.8 \pm 4.5$ & $77.7-96.0$ & $93.0 \pm 10.9$ & $67.5-124$ & $<0.001$ \\
\hline $\mathrm{MCH}(\mathrm{pg})$ & $30.4 \pm 3.30$ & $21.5-36.0$ & $29.6 \pm 2.3$ & $25.3-39.9$ & $29.9 \pm 3.9$ & $20.3-46.4$ & 0.63 \\
\hline $\mathrm{MCHC}(\mathrm{gm} / \mathrm{dl})$ & $35.6 \pm 2.90$ & $28.0-39.7$ & $33.4 \pm 1.3$ & $31.0-36.2$ & $32.2 \pm 3.4$ & $25.3-47.3$ & $<0.001$ \\
\hline RDW (\%CV) & $21.5 \pm 3.20$ & $15.4-26.9$ & $14.3 \pm 1.4$ & $11.8-17.4$ & $18.2 \pm 3.7$ & $12.0-31.9$ & $<0.001$ \\
\hline $\mathrm{WBC}\left(\times 10^{\wedge} 9 / 1\right)$ & $8.52 \pm 3.66$ & $2.94-18.34$ & $7.55 \pm 1.84$ & $4.01-11.36$ & $53.85 \pm 67.74$ & $3.23-367.25$ & $<0.001$ \\
\hline Platelets $\left(\times 10^{\wedge} 9 / 1\right)$ & $193 \pm 83$ & $3-331$ & $258 \pm 73$ & $140-404$ & $89 \pm 88$ & $5-481$ & $<0.001$ \\
\hline$\%$ Hyperchromic cells & $18.2 \pm 13.0$ & $6.3-50.9$ & $0.6 \pm 0.5$ & $0.1-2.4$ & $1.1 \pm 1.3$ & $0.0-8.9$ & $<0.001$ \\
\hline \multicolumn{8}{|l|}{ Flagging data } \\
\hline Hyperchromia flag & $21 / 21$ & & $0 / 50$ & & $1 / 50$ & & \\
\hline $\mathrm{HC}$ variance flag & $20 / 21$ & & $6 / 50$ & & $16 / 50$ & & \\
\hline
\end{tabular}


The RBC cytograms in all 21 patients showed variable number of RBCs plotted in the hyperchromic zone (Fig. 4: arrows). RBC cytograms in the control group did not show significant RBCs plotted in the hyperchromic zone. Only one patient with anemia due to other disorders showed an abnormal RBC cytogram similar to patients with HS. This was a 60 years old male with B cell lymphoma who had developed AIHA. Peripheral blood smear showed significant spherocytes and macrocytosis. Direct Coombs test was positive (3+).

\section{Discussion}

The present study has shown that automated hematology data like percentage of hyperchromic cells, hyperchromic cell flagging, and cytogram pattern of the RBC volume/hemoglobin concentration can be used to screen patients with suspected HS.

Although OFT is still used in the diagnosis of HS, its mild form is often difficult to identify clinically and may have a

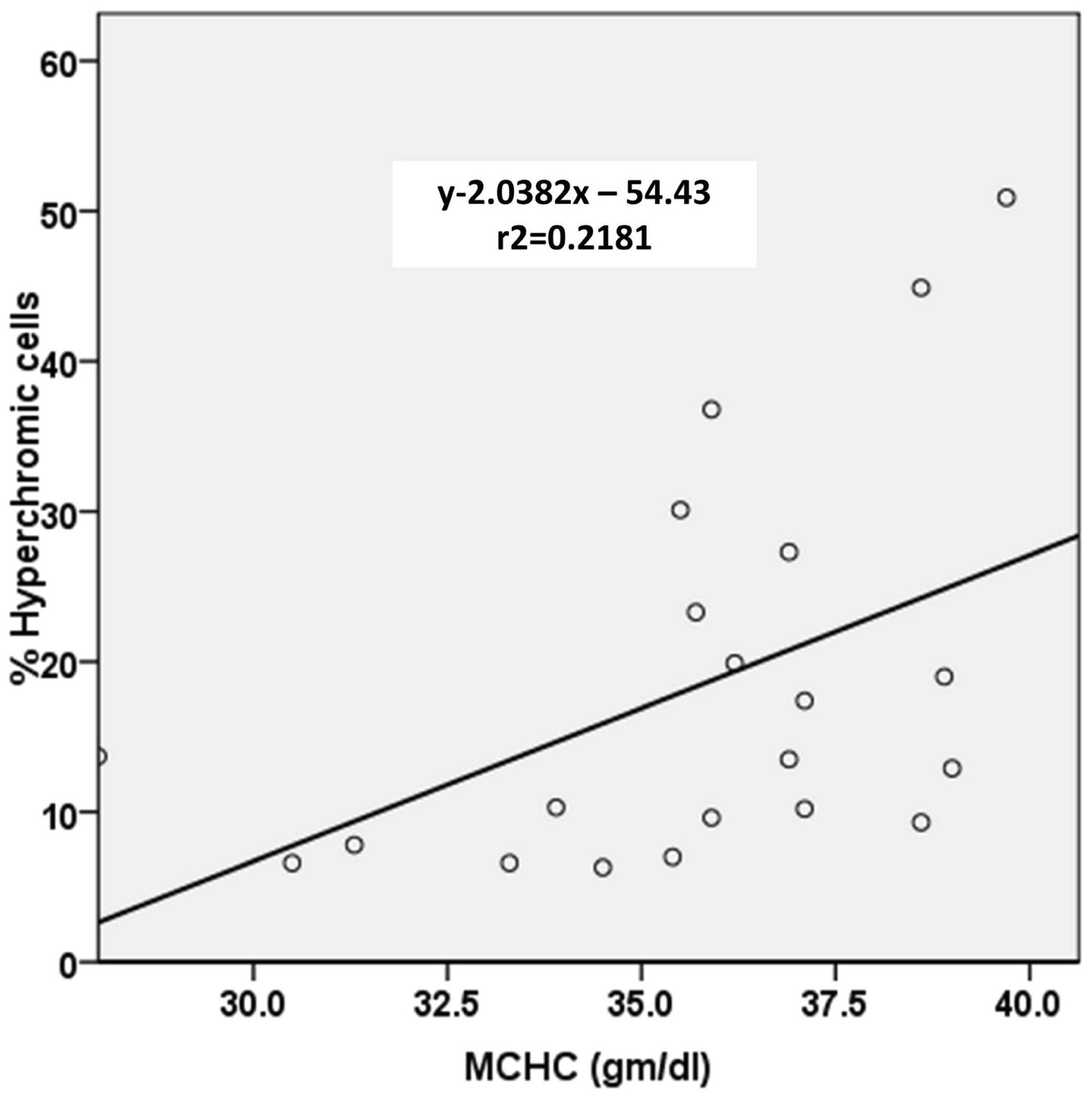

Fig. 3 Shows poor correlation between MCHC and percentage of hyperchromic cells $\left(r^{2}=0.2181\right)$ 
Fig. 4 RBC cytograms in 4 patients (a-d) with variable number $(6.3-50.9 \%)$ of spherocytes (arrows)
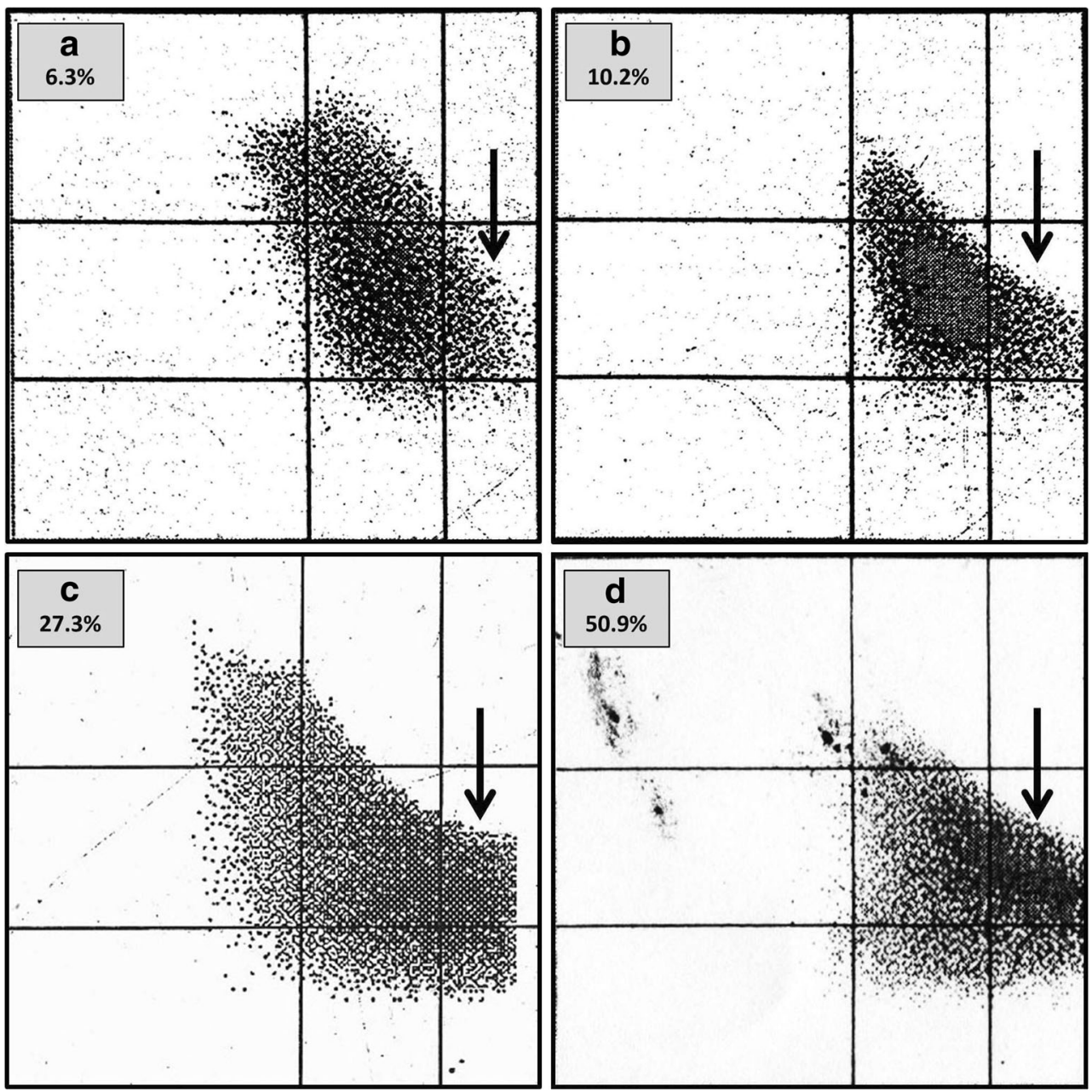

non-contributory OFT [18]. In approximately $20 \%$ of these, the diagnosis is missed [5]. In such cases, flow cytometric OFT and eosin-5'-maleimide dye-binding tests have a better sensitivity than conventional OFT $[19,20]$. Identification of the causative mutation, currently based on the whole-exome sequencing method, can provide a definite diagnosis. However, these techniques have limited availability and are expensive [3].

Previous studies have shown the utility of several novel RBC parameters generated by various automated hematology analyzers in screening patients with HS [10-17]. Rooney et al. assessed hyperchromic RBC count in 740 pediatric patients on the CELL-DYN Sapphire hematology analyzer. The number of hyperchromic RBCs was maximum in patients with HS (median 11.5\%; range 5.1-29.2\%). A 4.9\% cut-off for hyperchromic RBCs on the analyzer detected HS with $96.4 \%$ sensitivity and $99.1 \%$ specificity. The authors concluded that measurement of hyperchromic RBCs be used for rapid and cost-effective screening for HS [6].

In another study of 58 patients with HS, the authors found that \%Hyper cut-off of $6.4 \%$ showed a sensitivity of $92.3 \%$ and specificity of $90.7 \%$ to detect HS [20]. The minimum percentage of hyperchromic cells in patients with HS seen in our study was $6.3 \%$. A study on Technicon $\mathrm{H}^{*} 1$ analyzer assessed $\mathrm{CBC}$ in 21 patients with $\mathrm{HS}$ of which 9 patients were splenectomized; the authors concluded that the $\mathrm{RBC}$ cytogram appearances of HS were so specific that OFT could be easily avoided [11]. A study in 80 non-splenectomized patients of HS and 140 controls showed the percentage of hyperchromic cells and HDW to be significantly different in patients with HS $(p<0.001)$ [13].

In our study, the red cell volume/hemoglobin cytogram pattern showed a consistent pattern in all patients with HS in the form of a tail of cells in the hyperchromic zone. There was a significant difference $(p<0.0001)$ in the percentage of hyperchromic cells in patients with HS as compared to the control group.

A study in 34 patients with greater than $4 \%$ hyperchromic red cells concluded that subjects with an isolated increase in hyperchromic RBCs have RBC changes similar to that of patients with HS, but to a minor extent [10]. A study that screened $\mathrm{CBC}$ in 32,226 individuals showed that $162(0.5 \%)$ had more than $10 \%$ hyperchromic RBC. It was implied that if the percentage of hyperchromic RBCs is $>10 \%$, then it may 
be associated with both hereditary and acquired RBC membrane disorders, and extended follow-up must be done [21].

In our study, with an increasing number of spherocytes, the RBC cytogram showed more cells in the hyperchromic zone. The cytogram can give a quick visual estimate of the expected proportion of spherocytes in the peripheral blood smear. Also, in cases where the number of spherocytes are less and may be missed on a peripheral smear examination, the hyperchromia flagging and quantification of the cells may help the observer to detect the sparse spherocytes on a review of the blood smear. The cytograms can even indicate dual populations of red cell as can happen in decompensated HS which may show additional macrocytosis due to compensatory reticulocytosis. $\mathrm{RBC}$ indices alone in such cases may be misleading, and only $\mathrm{RBC}$ cytograms may indicate distinct cell populations. The cytogram may also provide a quick visual clue to the possibility of AIHA with very high-volume irregular RBC plots due to multiple RBCs being counted as one.

None of the samples from the control group showed hyperchromia flagging with only a minimal proportion of hyperchromic cells, well below the flagging cut-off of $4 \%$. Hypothetically, the cut-off would then also take care to exclude few aging RBCs in healthy individuals. Patients with other hematological disorders also did not show significant hyperchromic cells or abnormal RBC cytogram. The single patient who had significant number of hyperchromic cells was a 60-year-old male with autoimmune hemolytic anemia with a positive DCT. The rise in the percentage of hyperchromic cells in patients with HS in our study did not parallel an increase in MCHC. Thus, MCHC has limited utility as a screening tool, in the absence of a significant increase in the spherocyte numbers.

We emphasize that the numerical data and RBC cytogram would only assist in the identification of a spherocytic population and would not be a pointer to HS only. Acquired causes of spherocytosis like AIHA, transfusion reactions, snakebite, hyposplenism, and Rh-null phenotype are identified based on the clinical profile [5]. However, in patients with classical history and examination findings suggestive of HS, automated data can prove to be useful screening tool for identification and quantification of the spherocytic RBC population.

Our study highlights the potential role of hyperchromic cell percentage, flagging data, and RBC cytogram pattern. The data can be used as a quick screening tool in suspected cases of HS to guide confirmatory testing.

One of the limitations of this study is that although the analyzer flags indicate the presence of spherocytes in the blood sample, it cannot differentiate between autoimmune hemolytic anemia and hereditary spherocytosis. Although spherocytic cell population may be seen in cytograms, additional high-volume plots may not always be seen in patients with AIHA as the red cell autoagglutination may be inconsistent. Clinical profile and other ancillary investigations or confirmatory tests (DCT) are required to reach the final diagnosis. Also, the sample size in our study is limited. A larger cohort of patients with HS and other disorders needs to be studied for validation of our findings. The sensitivity and specificity figures proposed by us may be less if more numbers are studied.

\section{Conclusion}

The percentage of hyperchromic cells, its corresponding flags, and RBC cytogram pattern generated by Advia- 120 analyzer are a potential screening tool in patients with suspected HS.

Acknowledgments The study was conducted at the Christian Medical College \& Hospital, Ludhiana, Punjab, India.

Author's contributions MJJ examined the patients and did the clinical work up. NK reported the cases in the laboratory. S and NK collected and analyzed the numerical and graphical data. S, NK, and MJJ reviewed the literature and drafted the manuscript and take responsibility for the intellectual content. NK and MJJ did the statistical analysis. All authors have read and approved the final draft.

Data availability Yes, original instrument printouts are available for all cases.

\section{Compliance with ethical standards}

Conflict of interest The authors declare that they have no conflict of interest.

Ethics approval This retrospective chart review study involving human participants as part of routine care was in accordance with the ethical standards of the institutional and with the 1964 Helsinki Declaration and its later amendments or comparable ethical standards. The study has been approved by the Dean (Principal) of the Christian Medical College \& Hospital, Ludhiana, Punjab, India, where it was conducted.

Consent to participate Since this was retrospective series, no patient consent was taken.

Consent for publication Since this was retrospective series, no patient consent was taken. The generated data was a part of routine care.

Code availability Not applicable.

\section{References}

1. Eber SW, Pekrun A, Neufeldt A, Schröter W (1992) Prevalence of increased osmotic fragility of erythrocytes in German blood donors: screening using a modified glycerol lysis test. Ann Hematol 64:8892

2. Farias MG (2017) Advances in laboratory diagnosis of hereditary spherocytosis. Clin Chem Lab Med 55:944-948

3. Bolton-Maggs PH, Stevens RF, Dodd NJ, Lamont G, Tittensor P, King MJ (2004) Guidelines for the diagnosis and management of hereditary spherocytosis. Br J Haematol 126:455-474 
4. Korones D, Pearson HA (1989) Normal erythrocyte osmotic fragility in hereditary spherocytosis. J Pediatr 114:264-266

5. Gallagher PG, Glader B (2014) Hereditary spherocytosis, hereditary elliptocytosis, and other disorders associated with abnormalities of the erythrocyte membrane. In: Greer JP, Arber DA, Glader B, List AF, Means RT, Paraskevas F et al (eds) Wintrobe's clinical hematology, 13th edn. Lippincott Williams \& Wilkins, Philadelphia, pp 707-727

6. Rooney S, Hoffmann JJML, Cormack OM, McMahon C (2015) Screening and confirmation of hereditary spherocytosis in children using a CELL-DYN sapphire haematology analyser. Int J Lab Hematol 37:98-104

7. Manivannan P, Tyagi S, Chandra D, Mishra P, Pati HP, Saxena R (2019) Flow cytometric analysis of patients with hereditary spherocytosis - an Indian scenario. Hematology 23:175-180

8. He BJ, Liao L, Deng ZF, Tao YF, Xu YC, Lin FQ (2018) Molecular genetic mechanisms of hereditary spherocytosis: current perspectives. Acta Haematol 139:60-66

9. Sullivan E (2006) Hematology analyzer: from workhorse to thoroughbred. Lab Med 37:273-278

10. Conway AW, Vora AJ, Hinchliffe RF (2002) The clinical relevance of an isolated increase in the number of circulating hyperchromic red blood cells. J Clin Pathol 55:841-844

11. Pati AR, Patton WN, Harris RI (1989) The use of the Technicon H1 in the diagnosis of hereditary spherocytosis. Clin Lab Haematol 11: $27-30$

12. Ialongo P, Vignetti M, Cigliano G, Amadori S, Mandelli F (1989) Flow cytometric measurement (H-1 Technicon) of microcytic and hyperchromic red cell populations in pediatric patients affected by hereditary spherocytosis (HS). Haematologica 74:547-553

13. Ricard MP, Gilsanz F (1996) Assessment of the severity of hereditary spherocytosis using routine haematological data obtained with dual angle laser scattering cytometry. Clin Lab Haematol 18:75-78
14. Kutter D, Coulon N, Stirn F, Thoma M, Janecki J (2002) Demonstration and quantification of "hyperchromic" erythrocytes by haematological analysers. Application to screening for hereditary and acquired spherocytosis. Clin Lab 48:163-170

15. Michaels LA, Cohen AR, Zhao H, Raphael RI, Manno CS (1997) Screening for hereditary spherocytosis by use of automated erythrocyte indexes. J Pediatr 130:957-960

16. Cynober T, Mohandas N, Tchernia G (1996) Red cell abnormalities in hereditary spherocytosis: relevance to diagnosis and understanding of the variable expression of clinical severity. J Lab Clin Med 128:259-269

17. Mohandas N, Kim YR, Tycko DH, Orlik J, Wyatt J, Groner W (1986) Accurate and independent measurement of volume and hemoglobin concentration of individual red cells by laser light scattering. Blood 68:506-513

18. Delaunay J (2007) The molecular basis of hereditary red cell membrane disorders. Blood Rev 21:1-20

19. Arora RD, Dass J, Maydeo S, Arya V, Radhakrishnan N, Sachdeva A, Kotwal J, Bhargava M (2018) Flow cytometric osmotic fragility test and eosin-5'-maleimide dye-binding tests are better than conventional osmotic fragility tests for the diagnosis of hereditary spherocytosis. Int J Lab Hematol 40:335-342

20. Farias MG, Freitas PA (2015) Percentage of hyperdense cells: automated parameter to hereditary spherocytosis screening. Clin Biochem 48:1341-1343

21. Deuel JW, Lutz HU, Misselwitz B, Goede JS (2012) Asymptomatic elevation of the hyperchromic red blood cell subpopulation is associated with decreased red cell deformability. Ann Hematol 91: $1427-1434$

Publisher's note Springer Nature remains neutral with regard to jurisdictional claims in published maps and institutional affiliations. 